\title{
A note on a class of Hardy-Rellich type inequalities
}

Yanmei Di, Liya Jiang, Shoufeng Shen and Yongyang Jin*

"Correspondence:

yongyang@zjut.edu.cn

Department of Mathematics,

Zhejiang University of Technology,

Hangzhou, P.R. China

\begin{abstract}
In this note we provide simple and short proofs for a class of Hardy-Rellich type inequalities with the best constant, which extends some recent results.
\end{abstract}

MSC: 26D15; 35A23

Keywords: Hardy inequality; Hardy-Rellich inequality; Caffarelli-Kohn-Nirenberg inequality

\section{Introduction}

It is well known that Hardy's inequality and its generalizations play important roles in many areas of mathematics. The classical Hardy inequality is given by, for $N \geq 3$,

$$
\int_{R^{N}}|\nabla u(x)|^{2} d x \geq\left(\frac{N-2}{2}\right)^{2} \int_{R^{N}} \frac{|u(x)|^{2}}{|x|^{2}} d x
$$

where $u \in C_{0}^{\infty}\left(R^{N}\right)$, the constant $\left(\frac{N-2}{2}\right)^{2}$ is optimal and not attained.

Recently there has been a considerable interest in studying the Hardy-type and Rellichtype inequalities. See, for example, [1-7]. In [8] Caffarelli, Kohn and Nirenberg proved a rather general interpolation inequality with weights. That is the following so-called Caffarelli-Kohn-Nirenberg inequality. For any $u \in C_{0}^{\infty}\left(R^{N}\right)$, there exists $C>0$ such that

$$
\left\||x|^{\gamma} u\right\|_{L^{r}} \leq C\left\||x|^{\alpha}|\nabla u|\right\|_{L^{p}}^{a} \cdot\left\||x|^{\beta} u\right\|_{L^{q}}^{1-a}
$$

where

$$
\frac{1}{r}+\frac{\gamma}{N}=a\left(\frac{1}{p}+\frac{\alpha-1}{N}\right)+(1-a)\left(\frac{1}{q}+\frac{\beta}{N}\right)
$$

and

$$
\begin{aligned}
& p, q \geq 1, \quad r \geq 0, \quad 0 \leq a \leq 1, \quad \frac{1}{p}+\frac{\alpha}{N}>0, \quad \frac{1}{q}+\frac{\beta}{N}>0, \\
& \frac{1}{r}+\frac{\gamma}{N}>0, \quad \gamma=a \sigma+(1-a) \beta .
\end{aligned}
$$

In [9] Costa proved the following $L^{2}$-case version for a class of Caffarelli-KohnNirenberg inequalities with a sharp constant by an elementary method. For all $a, b \in R$

\section{Springer}

(c) 2013 Di et al.; licensee Springer. This is an Open Access article distributed under the terms of the Creative Commons Attribution License (http://creativecommons.org/licenses/by/2.0), which permits unrestricted use, distribution, and reproduction in any medium, provided the original work is properly cited. 
and $u \in C_{0}^{\infty}\left(R^{N} \backslash\{0\}\right)$,

$$
\hat{C} \int_{R^{N}} \frac{|u|^{2}}{|x|^{a+b+1}} d x \leq\left(\int_{R^{N}} \frac{|u|^{2}}{|x|^{2 a}} d x\right)^{\frac{1}{2}}\left(\int_{R^{N}} \frac{|\nabla u|^{2}}{|x|^{2 b}} d x\right)^{\frac{1}{2}},
$$

where the constant $\hat{C}=\hat{C}(a, b):=\frac{|N-(a+b+1)|}{2}$ is sharp.

On the other hand, the Rellich inequality is a generalization of the Hardy inequality to second-order derivatives, and the classical Rellich inequality in $R^{N}$ states that for $N \geq 5$ and $u \in C_{0}^{\infty}\left(R^{N} \backslash\{0\}\right)$

$$
\int_{R^{N}}|\Delta u(x)|^{2} d x \geq\left(\frac{N(N-4)}{4}\right)^{2} \int_{R^{N}} \frac{|u(x)|^{2}}{|x|^{4}} d x .
$$

The constant $\frac{N^{2}(N-4)^{2}}{16}$ is sharp and never achieved. In [10] Tetikas and Zographopoulos obtained a corresponding stronger versions of the Rellich inequality which reads

$$
\left(\frac{N}{2}\right)^{2} \int_{R^{N}} \frac{|\nabla u|^{2}}{|x|^{2}} d x \leq \int_{R^{N}}|\Delta u|^{2} d x
$$

for all $u \in C_{0}^{\infty}$ and $N \geq 3$. In [11] Costa obtained a new class of Hardy-Rellich type inequalities which contain (1.5) as a special case. If $a+b+3 \leq N$, then

$$
\hat{C} \int_{R^{N}} \frac{|\nabla u|^{2}}{|x|^{a+b+1}} d x \leq\left(\int_{R^{N}} \frac{|\Delta u|^{2}}{|x|^{2 b}} d x\right)^{\frac{1}{2}}\left(\int_{R^{N}} \frac{|\nabla u|^{2}}{|x|^{2 a}} d x\right)^{\frac{1}{2}},
$$

where the constant $\hat{C}=\hat{C}(a, b):=\left|\frac{N+a+b-1}{2}\right|$ is sharp.

The goal of this paper is to extend the above (1.3) and (1.6) to the general $L^{p}$ case for $1<p<\infty$ by a different and direct approach.

\section{Main results}

In this section, we will give the proof of the main theorems.

Theorem 1 For all $a, b \in R$ and $u \in C_{0}^{\infty}\left(R^{N} \backslash\{0\}\right)$, one has

$$
C \int_{R^{N}} \frac{|u|^{p}}{|x|^{a+b+1}} d x \leq\left(\int_{R^{N}} \frac{|\nabla u|^{p}}{|x|^{a p}} d x\right)^{\frac{1}{p}}\left(\int_{R^{N}} \frac{|u|^{p}}{|x|^{b \frac{p}{p-1}}} d x\right)^{\frac{p-1}{p}},
$$

where $1<p<\infty$ and the constant $C=\left|\frac{N-(a+b+1)}{p}\right|$ is sharp.

Proof Let $u \in C_{0}^{\infty}\left(R^{N} \backslash\{0\}\right), a, b \in R$ and $\lambda=a+b+1$. By integration by parts and the Hölder inequality, one has

$$
\begin{aligned}
\int_{R^{N}} \frac{|u|^{p}}{|x|^{\lambda}} d x & =\frac{1}{N-\lambda} \int_{R^{N}}|u|^{p} \operatorname{div}\left(\frac{x}{|x|^{\lambda}}\right) d x \\
& =-\frac{1}{N-\lambda} \int_{R^{N}} p u|u|^{p-2} \frac{x \cdot \nabla u}{|x|^{\lambda}} d x \\
& \leq\left|\frac{-p}{N-\lambda}\right| \int_{R^{N}} \frac{|x \cdot \nabla u|}{|x|^{\lambda}}|u|^{p-1} d x
\end{aligned}
$$




$$
\begin{aligned}
& \leq\left|\frac{p}{N-\lambda}\right| \int_{R^{N}} \frac{|\nabla u||u|^{p-1}}{|x|^{a+b}} d x \\
& \leq\left|\frac{p}{N-\lambda}\right|\left(\int_{R^{N}} \frac{|\nabla u|^{p}}{|x|^{a p}} d x\right)^{\frac{1}{p}}\left(\int_{R^{N}} \frac{|u|^{p}}{|x|^{\frac{p}{p-1}}} d x\right)^{\frac{p-1}{p}} .
\end{aligned}
$$

Then

$$
\left|\frac{N-\lambda}{p}\right| \int_{R^{N}} \frac{|u|^{p}}{|x|^{\lambda}} d x \leq\left(\int_{R^{N}} \frac{|\nabla u|^{p}}{|x|^{a p}} d x\right)^{\frac{1}{p}}\left(\int_{R^{N}} \frac{|u|^{p}}{|x|^{\frac{p}{p-1}}} d x\right)^{\frac{p-1}{p}} .
$$

It remains to show the sharpness of the constant. By the condition with equality in the Hölder inequality, we consider the following family of functions:

$$
u_{\varepsilon}(x)=e^{-\frac{C_{\varepsilon}}{\beta}|x|^{\beta}}, \quad \text { when } \beta=a-\frac{b}{p-1}+1 \neq 0
$$

and

$$
u_{\varepsilon}(x)=\frac{1}{|x|^{C_{\varepsilon}}}, \quad \text { when } \beta=a-\frac{b}{p-1}+1=0
$$

where $C_{\varepsilon}$ is a positive number sequence converging to $\left|\frac{N-(a+b+1)}{p}\right|$ as $\varepsilon \rightarrow 0$. By direct computation and the limit process, we know the constant $\frac{|N-(a+b+1)|}{p}$ is sharp.

Remark 1 When $p=2$, the inequality (2.1) covers the inequality (2.4) in [9].

Remark 2 When $a=0, b=p-1$, the inequality (2.1) is the classical $L^{p}$ Hardy inequality:

$$
\left(\frac{N-p}{p}\right)^{p} \int_{R^{N}} \frac{|u|^{p}}{|x|^{p}} d x \leq \int_{R^{N}}|\nabla u|^{p} d x
$$

When we take special values for $a, b$, the following corollary holds.

Corollary 1 (i) When $b=(a+1)(p-1)$, the inequality (2.1) is just the weighted Hardy inequality:

$$
\left|\frac{N-p(a+1)}{p}\right|^{p} \int_{R^{N}} \frac{|u|^{p}}{|x|^{(a+1) p}} d x \leq \int_{R^{N}} \frac{|\nabla u|^{p}}{|x|^{a p}} d x .
$$

(ii) When $a+b+1=a p$, according to the inequality (2.1), we have

$$
\left|\frac{N-a p}{p}\right| \int_{R^{N}} \frac{|u|^{p}}{|x|^{a p}} d x \leq\left(\int_{R^{N}} \frac{|\nabla u|^{p}}{|x|^{a p}} d x\right)^{\frac{1}{p}}\left(\int_{R^{N}} \frac{|u|^{p}}{|x|^{a p-\frac{p}{p-1}}} d x\right)^{\frac{p-1}{p}}
$$

(iii) When $a=-p$ and $a+b+1=0$, we obtain the inequality

$$
\frac{N}{p} \int_{R^{N}}|u|^{p} d x \leq\left(\int_{R^{N}}|\nabla u|^{p}|x|^{p^{2}} d x\right)^{\frac{1}{p}}\left(\int_{R^{N}} \frac{|u|^{p}}{|x|^{p}} d x\right)^{\frac{p-1}{p}} .
$$


Di et al. Journal of Inequalities and Applications 2013, 2013:84

Page 4 of 6

http://www.journalofinequalitiesandapplications.com/content/2013/1/84

By a similar method, we can prove the following $L^{p}$ case Hardy-Rellich type inequality.

Theorem 2 Let $1<p<N, \frac{p-N}{p-1} \leq a+b+1 \leq 0$. Then, for any $u \in C_{0}^{\infty}\left(R^{N} \backslash\{0\}\right)$, the following holds:

$$
\hat{C} \int_{R^{N}} \frac{|\nabla u|^{p}}{|x|^{a+b+1}} d x \leq\left(\int_{R^{N}} \frac{\left|\triangle_{p} u\right|^{p}}{|x|^{a p}} d x\right)^{\frac{1}{p}}\left(\int_{R^{N}} \frac{|\nabla u|^{q}}{|x|^{b q}} d x\right)^{\frac{1}{q}}
$$

where $\frac{1}{p}+\frac{1}{q}=1, \hat{C}=\left(\frac{N-p+(p-1)(a+b+1)}{p}\right)$ and $\triangle_{p} u=\operatorname{div}\left(|\nabla u|^{p-2} \nabla u\right)$ is the $p$-Laplacian operator.

Proof Set $\lambda=a+b+1$, it is easy to see

$$
\begin{aligned}
\int_{R^{N}} \frac{|\nabla u|^{p}}{|x|^{\lambda}} d x & =\frac{1}{N-\lambda} \int_{R^{N}}|\nabla u|^{p} \operatorname{div}\left(\frac{x}{|x|^{\lambda}}\right) d x \\
& =-\frac{1}{N-\lambda} \int_{R^{N}} \frac{p}{2}|\nabla u|^{p-2} \frac{x}{|x|^{\lambda}} \cdot \nabla\left(|\nabla u|^{2}\right) d x \\
& =\frac{p}{2(\lambda-N)} \int_{R^{N}}|\nabla u|^{p-2} \frac{x \cdot \nabla\left(|\nabla u|^{2}\right)}{|x|^{\lambda}} d x .
\end{aligned}
$$

On the other hand,

$$
\begin{aligned}
\int_{R^{N}} \triangle_{p} u \frac{x \cdot \nabla u}{|x|^{\lambda}} d x & =\int_{R^{N}} \operatorname{div}\left(|\nabla u|^{p-2} \nabla u\right) \frac{x \cdot \nabla u}{|x|^{\lambda}} d x \\
& =-\int_{R^{N}}|\nabla u|^{p-2} \nabla u \cdot \nabla\left(\frac{x \cdot \nabla u}{|x|^{\lambda}}\right) d x \\
& =-\int_{R^{N}}|\nabla u|^{p-2}\left(\frac{|\nabla u|^{2}}{|x|^{\lambda}}+\frac{\frac{1}{2} x \cdot \nabla\left(|\nabla u|^{2}\right)}{|x|^{\lambda}}-\lambda \frac{(x \cdot \nabla u)^{2}}{|x|^{\lambda+2}}\right) d x
\end{aligned}
$$

which means

$$
\begin{aligned}
& \int_{R^{N}}|\nabla u|^{p-2} \cdot \frac{x \cdot \nabla\left(|\nabla u|^{2}\right)}{|x|^{\lambda}} d x \\
& \quad=2\left(\lambda \int_{R^{N}}|\nabla u|^{p-2} \frac{(x \cdot \nabla u)^{2}}{|x|^{\lambda}} d x-\int_{R^{N}} \frac{|\nabla u|^{p}}{|x|^{\lambda}} d x-\int_{R^{N}} \triangle_{p} u \frac{x \cdot \nabla u}{|x|^{\lambda}}\right) .
\end{aligned}
$$

Then, we can deduce from (2.8) and (2.9)

$$
\begin{aligned}
& \int_{R^{N}} \frac{|\nabla u|^{p}}{|x|^{\lambda}} d x \\
& \quad=\frac{p}{\lambda-N}\left(\lambda \int_{R^{N}}|\nabla u|^{p-2} \frac{(x \cdot \nabla u)^{2}}{|x|^{\lambda}} d x-\int_{R^{N}} \frac{|\nabla u|^{p}}{|x|^{\lambda}} d x-\int_{R^{N}} \triangle_{p} u \frac{x \cdot \nabla u}{|x|^{\lambda}}\right) .
\end{aligned}
$$

That is,

$$
\frac{N-p-\lambda}{p} \int_{R^{N}} \frac{|\nabla u|^{p}}{|x|^{\lambda}} d x+\lambda \int_{R^{N}}|\nabla u|^{p-2} \frac{(x \cdot \nabla u)^{2}}{|x|^{\lambda+2}} d x=\int_{R^{N}} \Delta_{p} u \frac{x \cdot \nabla u}{|x|^{\lambda}} d x .
$$


By the Hölder inequality,

$$
\int_{R^{N}} \triangle_{p} u \frac{x \cdot \nabla u}{|x|^{\lambda+2}} d x \leq\left(\int_{R^{N}} \frac{\left|\triangle_{p} u\right|^{q}}{|x|^{a q}}\right)^{\frac{1}{q}}\left(\int_{R^{N}} \frac{|\nabla u|^{p}}{|x|^{b p}}\right)^{\frac{1}{p}}
$$

note that $\frac{p-N}{p-1} \leq \lambda \leq 0$. Thus

$$
\frac{N-p+(p-1) \lambda}{p} \int_{R^{N}} \frac{|\nabla u|^{p}}{|x|^{\lambda}} d x \leq\left(\int_{R^{N}} \frac{\left|\triangle_{p} u\right|^{p}}{|x|^{a p}}\right)^{\frac{1}{p}}\left(\int_{R^{N}} \frac{|\nabla u|^{q}}{|x|^{b q}}\right)^{\frac{1}{q}} .
$$

We mention that we do not know whether the constant $\left(\frac{N-p+(p-1)(a+b+1)}{p}\right)$ in $(2.7)$ is optimal or not.

Corollary 2 When $a+b+1=0$, we have the following inequalities:

(i) when $a=-1, b=0$, the inequality (2.7) is equivalent to the inequality

$$
\left(\frac{N-p}{p}\right)^{p} \int_{R^{N}}|\nabla u|^{p} d x \leq \int_{R^{N}}\left|\triangle_{p} u\right|^{p}|x|^{p} d x .
$$

(ii) When $a=1, b=-2$, we obtain the inequality

$$
\left(\frac{N-p}{p}\right) \int_{R^{N}}|\nabla u|^{p} d x \leq\left(\int_{R^{N}} \frac{\left|\triangle_{p} u\right|^{p}}{|x|^{p}} d x\right)^{\frac{1}{p}}\left(\int_{R^{N}}|\nabla u|^{q}|x|^{2 q} d x\right)^{\frac{1}{q}}
$$

(iii) When $a=0, b=-1$, we get

$$
\left(\frac{N-p}{p}\right) \int_{R^{N}}|\nabla u|^{p} d x \leq\left(\int_{R^{N}}\left|\triangle_{p} u\right|^{p} d x\right)^{\frac{1}{p}}\left(\int_{R^{N}}|\nabla u|^{q}|x|^{q} d x\right)^{\frac{1}{q}} .
$$

\section{Competing interests}

The authors declare that they have no competing interests.

\section{Authors' contributions}

All authors jointly worked on the results and they read and approved the final manuscript.

\section{Acknowledgements}

This work is supported by NNSF of China (11001240), ZJNSF (LQ12A01023) and the foundation of the Zhejiang University of the Technology (20100229).

Received: 31 May 2012 Accepted: 18 February 2013 Published: 4 March 2013

\section{References}

1. Adimurthi, AS: Role of the fundamental solution in Hardy-Sobolev type inequalities. Proc. R. Soc. Edinb., Sect. A 136, 1111-1130 (2006)

2. Garofalo, N, Lanconelli, E: Frequency functions on the Heisenberg group, the uncertainty principle and unique continuation. Ann. Inst. Fourier (Grenoble) 40, 313-356 (1990)

3. Goldstein, JA, Kombe, I: Nonlinear degenerate parabolic equations on the Heisenberg group. Int. J. Evol. Equ. 1, 1-22 (2005)

4. Goldstein, JA, Zhang, QS: On a degenerate heat equation with a singular potential. J. Funct. Anal. 186, 342-359 (2001)

5. Jin, Y, Han, Y: Weighted Rellich inequality on $\mathrm{H}$-type groups and nonisotropic Heisenberg groups. J. Inequal. Appl. 2010, Article ID 158281 (2010)

6. Jin, Y, Zhang, G: Degenerate $p$-Laplacian operators and Hardy type inequalities on h-type groups. Can. J. Math. 62 , 1116-1130 (2010)

7. García Azorero, JP, Peral Alonso, I Hardy inequalities and some critical elliptic and parabolic problems. J. Differ. Equ. 144, 441-476 (1998) 
8. Caffarelli, L, Kohn, R, Nirenberg, L: First order interpolation inequalities with weights. Compos. Math. 53, 259-275 (1984)

9. Costa, DG: Some new and short proofs for a class of Caffarelli-Kohn-Nirenberg type inequalities. J. Math. Anal. Appl. 337, 311-317 (2008)

10. Tertikas, A, Zographopoulos, NB: Best constants in the Hardy-Rellich inequalities and related improvements. Adv. Math. 209, 407-459 (2007)

11. Costa, DG: On Hardy-Rellich type inequalities in $R^{N}$. Appl. Math. Lett. 22, $902-905$ (2009)

doi:10.1186/1029-242X-2013-84

Cite this article as: Di et al.: A note on a class of Hardy-Rellich type inequalities. Journal of Inequalities and Applications 2013 2013:84

Submit your manuscript to a SpringerOpen ${ }^{\odot}$ journal and benefit from:

- Convenient online submission

- Rigorous peer review

- Immediate publication on acceptance

- Open access: articles freely available online

- High visibility within the field

- Retaining the copyright to your article

Submit your next manuscript at $>$ springeropen.com 